\title{
BMJ Open Changing trends of HIV, syphilis, HCV infections and behavioural factors among female sex workers in Chongqing, China: findings from six serial surveillance surveys
}

\author{
Ling Hu, ${ }^{1}$ Guohui Wu, ${ }^{2}$ Rongrong Lu, ${ }^{2}$ Hua Zhu, ${ }^{1}$ Hongfang Qiu, ${ }^{1}$ Dan Jing, ${ }^{1}$ \\ Mengliang $\mathrm{Ye}$ (i) ${ }^{1}$
}

To cite: Hu L, Wu G, Lu R, et al. Changing trends of HIV, syphilis, $\mathrm{HCV}$ infections and behavioural factors among female sex workers in Chongqing, China: findings from six serial surveillance surveys. BMJ Open 2020;10:e036654. doi:10.1136/ bmjopen-2019-036654

- Prepublication history for this paper is available online. To view these files, please visit the journal online (http://dx.doi. org/10.1136/bmjopen-2019036654).

LH and GW contributed equally.

Received 24 December 2019 Revised 11 September 2020 Accepted 15 September 2020

Check for updates

(c) Author(s) (or their employer(s)) 2020. Re-use permitted under CC BY-NC. No commercial re-use. See rights and permissions. Published by BMJ.

${ }^{1}$ Department of Epidemiology and Health Statistics, School of Public Health and Management, Chongqing Medical University, Chongqing, China

${ }^{2}$ Department of AIDS/STD

Control and Prevention,

Chongqing Center for Disease

Control and Prevention,

Chongqing, China

Correspondence to

Dr Mengliang Ye;

yemengliang@cqmu.edu.cn

\section{ABSTRACT}

Objectives To explore the prevalence and changing trends of HIV, syphilis, hepatitis C virus (HCV) infections and risk behaviours among female sex workers (FSWs) and to provide reference and theoretical basis for formulating targeted interventions.

Design Six consecutive cross-sectional surveys. Setting Chongqing, China.

Participants FSWs were included if they (1) were aged $\geq 16$ years, (2) provided commercial sex for money or goods during the previous month and (3) were willing to participate in the survey and could provide verbal informed consent. This study included 16791 of 16810 participants recruited between 2013 and 2018.

Primary and secondary outcome measures The prevalence of HIV/syphilis/HCV infection.

Results The HIV and HCV prevalence among FSWs in Chongqing was stable during the study period, but the prevalence of syphilis had an increasing trend, particularly among low-tier and middle-tier FSWs. Improvements in HIV-related knowledge, condom use, injecting drug use and participation in HIV-related services were observed. However, no change was found in the prevalence of drug use. HIV infection was correlated with no condom use in the last commercial sex (adjusted OR (aOR) 3.48, $95 \% \mathrm{Cl} 1.90$ to 6.37 ) and syphilis infection (aOR 4.88, $95 \% \mathrm{Cl} 1.95$ to 12.18). Syphilis infection was correlated with inconsistent condom use (aOR 1.30, 95\% $\mathrm{Cl} 1.02$ to 1.65), HIV infection (aOR 5.88, $95 \% \mathrm{Cl} 2.40$ to 14.41 ), HCV infection (aOR 7.68, 95\% Cl 4.37 to 13.49) and sexually transmitted infection (STI) diagnosis in the past year (aOR $3.81,95 \% \mathrm{Cl} 2.40$ to 6.03 ). HCV infection was associated with injecting drug use (aOR 8.91, 95\% Cl 4.45 to 17.86) and syphilis infection (aOR $7.88,95 \% \mathrm{Cl} 4.49$ to 13.83).

Conclusions Comprehensive interventions targeting FSWs, particularly low-tier and middle-tier FSWs, should be increasingly implemented to prevent and control HIV, syphilis and other STIs.

\section{INTRODUCTION}

The HIV/AIDS pandemic, one of the major public health problems all over the world, is highly heterogeneous among different
Strengths and limitations of this study

- The study involved 6 consecutive years of sentinel surveillance among female sex workers (FSWs) in Chongqing with a large sample size.

- This study provides valuable information about the prevalence and trends of sexually transmitted infections among FSWs in Chongqing, and helps to formulate and improve relevant intervention strategies.

- However, causality could not be determined due to the cross-sectional design.

- Recall bias and social desirability bias may be present due to the use of self-reported measures of condom use, drug use and other behaviour.

populations and regions. Compared with adult women, female sex workers (FSWs) bear a higher burden of HIV, with an estimated HIV prevalence of $10.4 \%$ (95\% CI $9.5 \%$ to $11.5 \%){ }^{1}$ They are also considered one of the most vulnerable groups to the acquisition of other sexually transmitted infections (STIs), such as syphilis. $^{2}$

After the implementation of reform and opening up in the late 1970s, the sex industry has re-emerged in China due to economic growth, population migration and changes in sexual attitudes. ${ }^{34}$ A large number of rural migrants moved to cities for enhanced job opportunities. However, those rural women found that the labour force was saturated or the wages were not high enough; then, sex work with quick financial return became an option. ${ }^{5}$ The estimated number of FSWs was 4 million $^{6}$; and approximately $6.9 \%$ of men aged 18-49 years have had commercial sex during their lifetime. ${ }^{7}$ The incidence of STIs, such as syphilis, has dramatically increased along with the prosperity of the sex trade. ${ }^{89}$ Heterosexual sex has replaced intravenous drug use and commercial 
blood/plasma collection as the primary transmission mode of HIV in China. ${ }^{10}$ Among the newly reported HIV infections in January-October 2019, heterosexual transmission accounted for $73.7 \%$, much higher than other transmission routes. ${ }^{11}$ A review also reported that FSWs in China had a median HIV prevalence of $0.6 \%(0 \%$ to $10.3 \%)$ and a rate of positive for at least one STI of $41.5 \%$ (13\% to $90.6 \%) .{ }^{12}$

As a developing country with a population of 1.4 billion, China faces more challenges in responding to the AIDS and STIs epidemic. The prevalence varies from province to province and Southwest provinces have a higher prevalence of STI. ${ }^{13} 14$ For example, although HIV prevalence among FSWs was lower than $0.05 \%$ in most provinces (such as Heilongjiang and Hebei) in 2012, it exceeded $1 \%$ in the same year in Yunnan and Guangxi. ${ }^{13}$ Furthermore, sex work is still illegal in China. Thus, it takes place in informal venues, including night clubs, hair salons, foot massage parlours and hotels, which is always combined with other works to cover up its illegality. This hinders the implementation of related interventions and reduces their effects. Different sex work settings are associated with the risk of HIV/STI infection. The average price of each sex transaction is often used by Chinese researchers to categorise FSWs into high, middle and low tiers. The size of different-tier FSWs may be related to the local economic level. In Jiangmen City, more than $60 \%$ of FSWs was high-tier FSW, while the proportion of low-tier FSWs was less than $10 \% .{ }^{15}$ However, in Jianshui County, the proportion of low-tier FSWs was close to $30 \% .{ }^{16}$ The proportion of low-tier FSWs may vary from region to region, but previous studies have shown that they have a higher risk of HIV infection than other FSWs. ${ }^{13} 17$

The Chinese government has provided voluntary HIV counselling and testing, STIs services, condom promotion and peer education among FSWs through the cooperation of Centers for Disease Control (CDC), community health services and medical institutions. ${ }^{18}$ The 'Four Frees and One Care' policy helps treat patients with AIDS who have financial difficulties by providing free antiviral drugs. In 1995, China established the national HIV sentinel surveillance system to actively monitor HIV prevalence among high-risk populations (eg, FSWs and injecting drug users) and guide the development of HIV prevention and control strategies. ${ }^{19-21}$ After years of development, the surveillance system has expanded its scope from 42 sentinel sites in 1995-1888 in 2010, and combined biological and behavioural surveillance strategies, which included serotesting for HIV, syphilis and hepatitis C virus (HCV) ${ }^{22}$ Surveillance data could help understand the epidemic status and trend of HIV and STIs among FSWs, which is necessary for developing HIV or STIs prevention programmes. ${ }^{23}$ According to China's surveillance data, the overall prevalence of HIV/syphilis/HCV among FSWs showed a downward trend from 2010 to $2015 .{ }^{24}$ However, there may be diverse trends in different geographic regions of China, and there has been less work on understanding trends of STIs among FSWs in underdeveloped southwest areas.
Chongqing, located in Southwest China, is one of the four Chinese municipalities directly controlled by the central government. It is geographically close to Yunnan and Guangxi and it has large population contacts with Yunnan and Guangxi. It is the political and economic centre of Western China and a city with obvious urbanrural dual structure and the prosperous sex industry. A study using the network scale-up method estimated that FSWs accounted for $0.4 \%$ of women aged 15-49 years in Chongqing, and the clients of FSWs represented 2\% of men aged 15-49 years. ${ }^{25}$ The HIV prevalence among FSWs in Chongqing in 2012 was estimated at $0.25 \%$, lower than that among other high-risk groups, such as men who have sex with men $(17.03 \%)$ and injecting drug users $(7.35 \%) .{ }^{26}$ However, FSWs can transmit STIs to numerous clients and then to the general population, which pose a great challenge to public health. Heterosexual transmission has become the main route of HIV transmission in Chongqing since 2007.27 Of the 6352 newly diagnosed HIV/AIDS cases reported in Chongqing in 2015, 75.08\% were infected through heterosexual transmission. ${ }^{27}$ Therefore, understanding the prevalence and trends of STIs among FSWs in Chongqing is highly important.

On the basis of the surveillance data in Chongqing, this study was aimed to identify the prevalence and changing trends of HIV, syphilis and HCV infections among FSWs over time. This study also explored the trends in sexual behaviours of FSWs and discussed the risk factors for prevalent STIs over a 6-year period in order to provide a reference for formulating interventions to control AIDS and STIs.

\section{METHODS}

\section{Study design}

Sentinel surveillance was conducted to collect HIV, syphilis and HCV prevalence and high-risk behaviour information through cross-sectional surveys. The data analysed in this study were sentinel surveillance data among FSWs in Chongqing from 2013 to 2018.

\section{Participant selection and data collection}

The detailed procedures of data collection followed the Operational Manual for the Implementation Program of National AIDS Sentinel Surveillance established by the National Center for AIDS Prevention and Control. ${ }^{28}$ As shown in figure 1 , serial cross-sectional surveys were conducted from April to June each year at seven sentinel surveillance sites in Chongqing (Yuzhong District, Jiulongpo District, Wanzhou District, Dianjiang, Hechuan, Qijiang (Wansheng) and Youyang). The sample size of each site was 400 participants. The entire sampling process went through the following three steps. First, a distribution map of known commercial workplaces in the monitoring area was drawn to construct a sampling frame and these locations were categorised into low-tier, middletier and high-tier venues on the basis of the average price of each sex transaction. Then, these venues in each city were randomly selected through proportional sampling. 


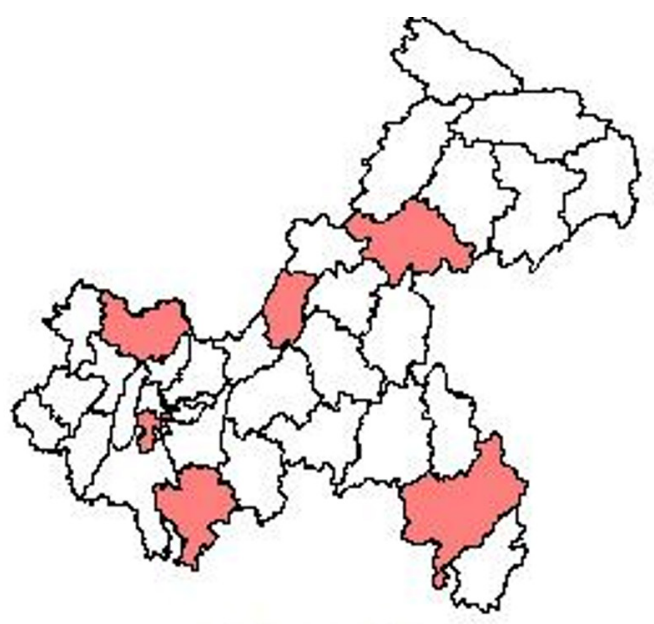

Chongqing

\section{Legend}

\section{Sentinel surveillance sites in Chongqing}

Figure 1 The location of seven sentinel surveillance sites in Chongqing.

The proportions of low-tier and middle-tier FSWs among all participants were at least $10 \%$ and $40 \%$, respectively. The FSWs were recruited if they met the inclusion criteria as follows: participants (1) were aged $\geq 16$ years, (2) provided commercial sex for money or goods during the previous month and (3) were willing to participate in the survey and could provide verbal informed consent.

All participants completed an anonymous, standard interviewer-administered and face-to-face questionnaire. After each interview, 3-5 mL of venous blood was collected from each participant for HIV, syphilis and HCV antibody detection. The blood sample was linked to the questionnaire by a unique identification code assigned to the participant.

\section{Measures}

Questionnaire

A questionnaire was used to collect the sociodemographic characteristics, HIV knowledge and behaviour of the FSWs.

1. Sociodemographic characteristics, including year of birth, marital status, household registration, ethnicity and education level were collected.

2. The HIV-related knowledge of participants was assessed using eight questions with 'yes', 'no' or 'don't know' as answers. The questions have been updated since 2016 and took new-type drugs and intentional transmission of HIV/AIDS into consideration. Only correct responses were scored as 1 point, whereas incorrect responses and 'don't know' responses did not earn any point. The Cronbach's alpha values were 0.706 and 0.739 before and after the questions were changed, respectively.

3. The participants' behavioural factors were also collected, including questions regarding condom use in last commercial sex, condom use with clients in the past month, drug use during their lifetime, STI diagnosis in the last year and participation in HIV-related services. In this study, consistent condom use (CCU) in the past month was defined as always using a condom during commercial intercourse.

\section{Laboratory testing}

In all surveys, the venous blood samples were tested for HIV, syphilis and HCV by trained laboratory technicians. Initial screening for HIV, syphilis and HCV antibodies was conducted using ELISA method (ELISA-1). When the result was negative, no further re-examination was carried out and infection status was recorded as negative. When the result was positive, HIV or HCV infection was confirmed by another ELISA method (ELISA-2) while syphilis infection was confirmed using a non-specific detection method called toluidine red unheated serum test. The results were determined as positive only when both tests were positive.

\section{Quality assurance}

All investigators were strictly trained before the survey to ensure that they familiarised the questionnaire structure and mastered the unified investigation standards and requirements. After the investigation, the investigators carefully reviewed the questionnaires and promptly corrected the missing items, wrong items and logical errors. Experts from the Chongqing Center for Disease Control and Prevention went to the investigation site for guidance and inspection to ensure quality. All laboratory tests were conducted at designated and certified laboratories in local CDC or hospitals.

\section{Data analysis}

The sociodemographic characteristics of participants were presented using descriptive statistics by survey year. In addition, the trends of HIV, syphilis, HCV infections and behavioural factors over time were assessed using Cochran-Armitage trend test. Multivariable logistic regression was conducted to identify related risk-factors of HIV, syphilis and HCV infection by using stepwise elimination. The ORs and 95\% CIs of each significant risk factor were also determined, unadjusted and adjusted for sociodemographic factors. All statistical analyses were carried out using Statistical Analysis Software, V.9.2 (SAS Institute).

\section{Patient and public involvement}

Patients and/or the public were not involved in the design, or conduct, or reporting, or dissemination plans of this research.

\section{RESULTS}

\section{Sociodemographic characteristics}

This study included 16791 of 16810 participants recruited between 2013 and 2018. Table 1 depicted all the participants' demographic characteristics stratified by year of survey. Nearly half of the participants (46.7\%) worked 
Table 1 Sociodemographic characteristics of female sex workers stratified by survey year (N, \%)

\begin{tabular}{|c|c|c|c|c|c|c|c|}
\hline & 2013 & 2014 & 2015 & 2016 & 2017 & 2018 & Total \\
\hline Characteristics & $\mathrm{N}(\%)$ & $\mathbf{N}(\%)$ & $\mathrm{N}(\%)$ & $\mathrm{N}(\%)$ & $\mathrm{N}(\%)$ & $\mathbf{N}(\%)$ & N (\%) \\
\hline Overall & 2793 (16.6) & 2796 (16.7) & 2793 (16.6) & 2799 (16.7) & 2805 (16.7) & 2805 (16.7) & $16791(100.0)$ \\
\hline \multicolumn{8}{|l|}{ Age group (years) } \\
\hline$<20$ & $285(10.2)$ & $226(8.1)$ & $206(7.4)$ & $228(8.1)$ & $87(3.1)$ & $96(3.4)$ & $1128(6.7)$ \\
\hline $20-45$ & $2404(86.1)$ & $2451(87.7)$ & $2442(87.4)$ & $2412(86.2)$ & $2521(89.9)$ & $2415(86.1)$ & $14645(87.2)$ \\
\hline$>45$ & $104(3.7)$ & $119(4.3)$ & $145(5.2)$ & $159(5.7)$ & $197(7.0)$ & $294(10.5)$ & $1018(6.1)$ \\
\hline \multicolumn{8}{|l|}{ Marital status } \\
\hline Never been married & $1131(40.5)$ & $1143(40.9)$ & $1102(39.5)$ & 1106 (39.5) & 734 (26.2) & $837(29.8)$ & $6053(36.0)$ \\
\hline Married & $1148(41.1)$ & $1104(39.5)$ & 1207 (43.2) & $1152(41.2)$ & $1279(45.6)$ & $1278(45.6)$ & $7168(42.7)$ \\
\hline Living together as if married & $275(9.8)$ & $308(11.0)$ & $260(9.3)$ & $307(11.0)$ & $305(10.9)$ & $296(10.6)$ & $1751(10.4)$ \\
\hline Divorced/widowed & 239 (8.6) & 241 (8.6) & $224(8.0)$ & 234 (8.4) & 487 (17.4) & $394(14.0)$ & 1819 (10.8) \\
\hline \multicolumn{8}{|l|}{ Household registration (hukou) } \\
\hline Chongqing & $2518(90.2)$ & 2559 (91.5) & $2533(90.7)$ & 2547 (91.0) & $2580(92.0)$ & $2537(90.4)$ & $15274(91.0)$ \\
\hline Other provinces & $275(9.8)$ & $237(8.5)$ & $260(9.3)$ & $252(9.0)$ & $225(8.0)$ & $268(9.6)$ & $1517(9.0)$ \\
\hline \multicolumn{8}{|l|}{ Ethnicity } \\
\hline Minority & $253(9.1)$ & $266(9.5)$ & $308(11.0)$ & 295 (10.5) & $280(10.0)$ & $272(9.7)$ & $1674(10.0)$ \\
\hline Han & 2539 (90.9) & $2530(90.5)$ & 2485 (89.0) & 2504 (89.5) & 2525 (90.0) & $2533(90.3)$ & $15116(90.0)$ \\
\hline \multicolumn{8}{|l|}{ Education level } \\
\hline Primary school or below & $512(18.3)$ & $524(18.7)$ & $581(20.8)$ & $506(18.1)$ & $595(21.2)$ & $507(18.1)$ & 3225 (19.2) \\
\hline Junior middle school & $1664(59.6)$ & $1570(56.2)$ & $1367(48.9)$ & 1509 (53.9) & 1635 (58.3) & $1446(51.6)$ & $9191(54.7)$ \\
\hline High school or above & $617(22.1)$ & $702(25.1)$ & $845(30.3)$ & $784(28.0)$ & $575(20.5)$ & $852(30.4)$ & $4375(26.1)$ \\
\hline \multicolumn{8}{|l|}{ Typology } \\
\hline Low-tier & $973(34.8)$ & 955 (34.2) & $1043(37.3)$ & $1193(42.6)$ & $1347(48.0)$ & $1161(41.4)$ & $6672(39.7)$ \\
\hline Middle-tier & $1347(48.2)$ & $1504(53.8)$ & $1448(51.8)$ & $1236(44.2)$ & $1192(42.5)$ & $1108(39.5)$ & $7835(46.7)$ \\
\hline High-tier & $473(16.9)$ & $337(12.1)$ & $302(10.8)$ & $370(13.2)$ & $266(9.5)$ & $536(19.1)$ & $2284(13.6)$ \\
\hline \multicolumn{8}{|l|}{ Local working time } \\
\hline More than 1 year & $651(23.3)$ & $566(20.2)$ & 751 (26.9) & $673(24.0)$ & $888(31.7)$ & $946(33.7)$ & $4475(26.7)$ \\
\hline $6-12$ months & $635(22.7)$ & $672(24.0)$ & $899(32.2)$ & $1018(36.4)$ & $918(32.7)$ & $833(29.7)$ & 4975 (29.6) \\
\hline $1-6$ months & $1097(39.3)$ & $1204(43.1)$ & $888(31.8)$ & $778(27.8)$ & $814(29.0)$ & $754(26.9)$ & 5535 (33.0) \\
\hline Less than 1 month & $409(14.6)$ & $354(12.7)$ & $255(9.1)$ & $330(11.8)$ & $185(6.6)$ & $272(9.7)$ & $1805(10.8)$ \\
\hline \multicolumn{8}{|l|}{ Location of previous job } \\
\hline Other provinces & $334(12.0)$ & $257(9.2)$ & $354(12.7)$ & $305(10.9)$ & $353(12.6)$ & $293(10.4)$ & $1896(11.3)$ \\
\hline Other cities in Chongqing & $330(11.8)$ & $569(20.4)$ & $549(19.7)$ & $572(20.4)$ & $356(12.7)$ & $352(12.5)$ & $2728(16.2)$ \\
\hline Current city & $1699(60.8)$ & $1627(58.2)$ & $1629(58.3)$ & $1576(56.3)$ & $1894(67.5)$ & $1872(66.7)$ & $10297(61.3)$ \\
\hline No previous work & $430(15.4)$ & $343(12.3)$ & $261(9.3)$ & $346(12.4)$ & $202(7.2)$ & $288(10.3)$ & $1870(11.1)$ \\
\hline \multicolumn{8}{|l|}{ HIV knowledge } \\
\hline$<6$ & $388(13.9)$ & $458(16.4)$ & $231(8.3)$ & 449 (16.0) & $348(12.4)$ & 305 (10.9) & 2179 (13.0) \\
\hline$\geq 6$ & 2405 (86.1) & 2338 (83.6) & 2562 (91.7) & $2350(84.0)$ & 2457 (87.6) & $2500(89.1)$ & $14612(87.0)$ \\
\hline
\end{tabular}

in middle-tier venues, $39.7 \%$ worked in low-tier venues and $13.6 \%$ worked in high-tier venues. The median age was 28 years and most $(87.2 \%)$ of them were between 20 and 45 years of age. The majority of the participants were in Chongqing households (91.0\%) and belong to Han $(90.0 \%)$. Less than one-third of them had high school or above education and half $(54.7 \%)$ had a junior middle school education. Most participants have worked in the current location for 1-6 months $(33.0 \%)$.

\section{Trends of HIV, syphilis and HCV prevalence}

During the study period, the overall prevalence of HIV, syphilis and HCV infection among the study population was $0.27 \%$ (95\% CI $0.19 \%$ to $0.35 \%), 1.73 \%$ (95\% CI $1.54 \%$ to $1.93 \%$ ) and $0.72 \%$ (95\% CI $0.59 \%$ to $0.85 \%$ ), respectively. Low-tier FSWs had higher prevalence of HIV, syphilis and HCV, with 6-year average prevalence rates of $0.40 \%, 2.53 \%$ and $1.08 \%$, respectively $(p=0.019$, $\mathrm{p}<0.001, \mathrm{p}<0.001)$. The HIV prevalence among FSWs in 


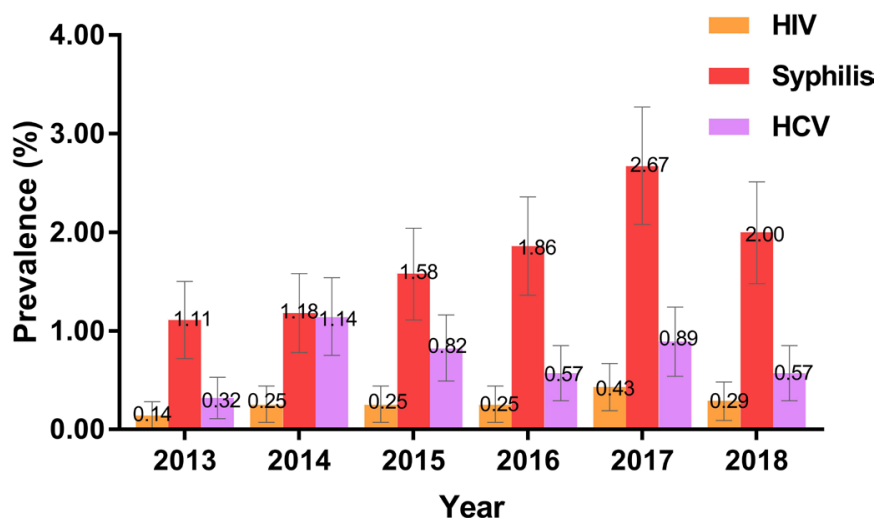

Figure 2 Prevalence of HIV, syphilis and HCV among FSWs in Chongqing by survey year. Bars show prevalence (per cent); error bars show 95\% Cls. FSWs, female sex workers; $\mathrm{HCV}$, hepatitis $\mathrm{C}$ virus.

Chongqing, which ranged from $0.14 \%$ to $0.43 \%$ during the study years, had no significant changes over time $(p=0.129$, figure 2$)$. No significant time trend was found among low-tier/middle-tier/high-tier FSWs $(\mathrm{p}=0.498$, 0.366 and 0.423 , respectively). The HCV prevalence among FSWs in Chongqing ranged from $0.32 \%$ to $1.14 \%$ between 2013 and 2018, with no significant trend. In each subgroup, no significant changing trend of HCV infection by year was found (figure 3). Unlike HIV and HCV, a total of $1.11 \%, 1.18 \%, 1.58 \%, 1.86 \%, 2.67 \%$ and $2.00 \%$ of the respondents were confirmed to be infected with syphilis from 2013 to 2018, showing an upward trend $(\mathrm{p}<0.001)$. This trend was also found in low-tier FSWs (from $1.13 \%$ in 2013 to $2.50 \%$ in 2018, $\mathrm{p}<0.001$ ) and middle-tier FSWs (from $1.19 \%$ in 2013 to $1.81 \%$ in $2018, \mathrm{p}=0.026$ ), but not in high-tier FSWs $(p=0.713)$.

\section{Changing trends of behavioural factors}

Of the whole survey population, more than $80 \%$ exhibited HIV-related knowledge and used condom in their last commercial sex (table 2). Nearly $90 \%$ received at least one of the following three HIV-related services in the last year: (1) condom promotion and distribution and AIDS counselling and testing, (2) community drug maintenance therapy and clean needle supply/exchange and (3) peer education. In addition, about half of the participants used condom consistently with clients in the last month. Approximately 3\% reported drug use, and 2\% reported STI diagnosis in the past year.

The changing trends of FSWs' behaviours were also examined, and results showed no significant change over time in drug use reported by FSWs. However, significant improvements over time were found in HIV-related knowledge, condom use, participation in HIV-related services and self-reported STI diagnosis in the last year among the FSWs.

\section{Correlates of HIV, syphilis and HCV infections}

Multivariable logistic regression analysis results (table 3) showed that, (1) HIV infection was significantly and positively associated with no condom use in the last commercial sex (adjusted OR (aOR) 3.48, 95\% CI 1.90 to 6.37) and syphilis infection (aOR 4.88, 95\% CI 1.95 to 12.18); (2) syphilis infection was significantly and positively associated with inconsistent condom use in the past month (aOR $1.30,95 \%$ CI 1.02 to 1.65 ), STI diagnosis in the past year (aOR 3.81, 95\% CI 2.40 to 6.03), HIV infection (aOR $5.88,95 \%$ CI 2.40 to 14.41 ) and HCV infection (aOR 7.68, 95\% CI 4.37 to 13.49 ) and (3) HCV infection was significantly and positively associated with injecting drug use (aOR $8.91,95 \%$ CI 4.45 to 17.86 ) and syphilis infection (aOR $7.88,95 \%$ CI 4.49 to 13.83 ).

\section{DISCUSSION}

FSWs are considered to be at high risk of HIV infection and other STIs and contribute disproportionately to the transmission. Surveillance of STIs and risk behaviour among FSWs is highly important for the understanding of the potential of spreading HIV to the general population and the assessment and improvement of interventions targeted at FSWs. ${ }^{23}$ Although analysing and knowing the prevalence and changing trends of HIV, syphilis, HCV and relevant behaviours among FSWs are critical, only few studies have been reported in Chongqing, China. Thus, in the present study, the prevalence and changing trends of HIV, syphilis, HCV and behaviour of Chongqing FSWs were examined using sentinel surveillance data from 2013 to 2018.

The results showed that the HIV prevalence among FSWs in Chongqing was relatively stable, with an annual average of $0.27 \%$ from 2013 to 2018, similar to $0.22 \%$
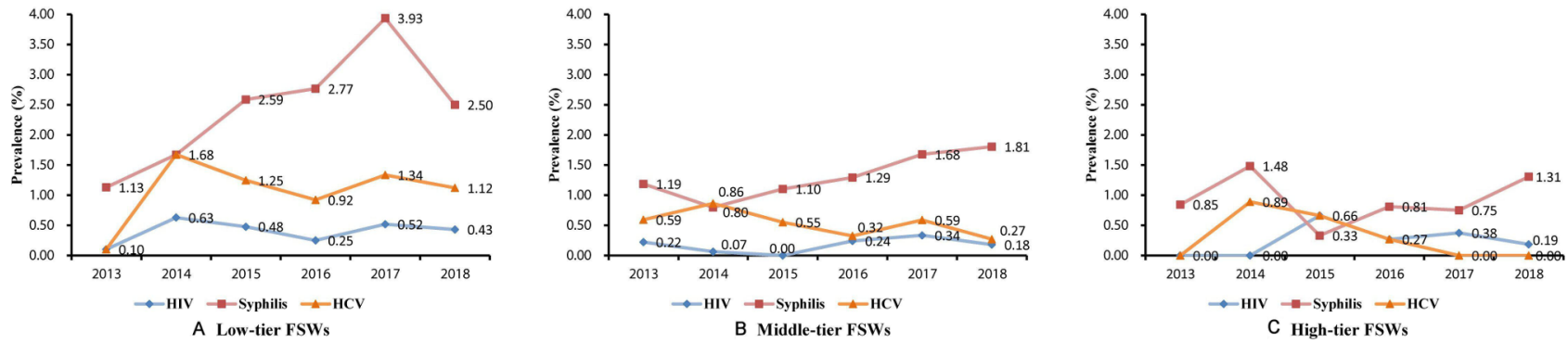

Figure 3 Prevalence of HIV, syphilis and HCV among low-tier FSWs (A), middle-tier FSWs (B) and high-tier FSWs (C) in Chongqing by survey year. (A) Low-tier FSWs; (B) middle-tier FSWs; (C) high-tier FSWs. FSWs, female sex workers; HCV, hepatitis $C$ virus. 


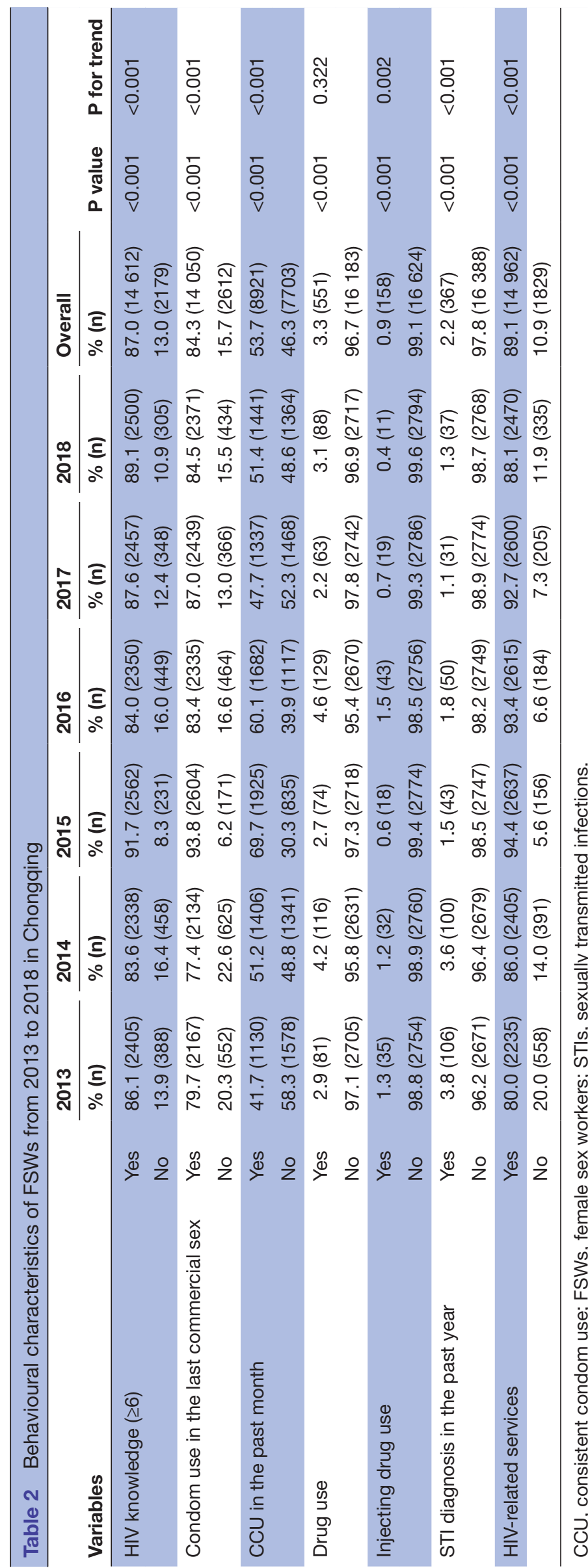




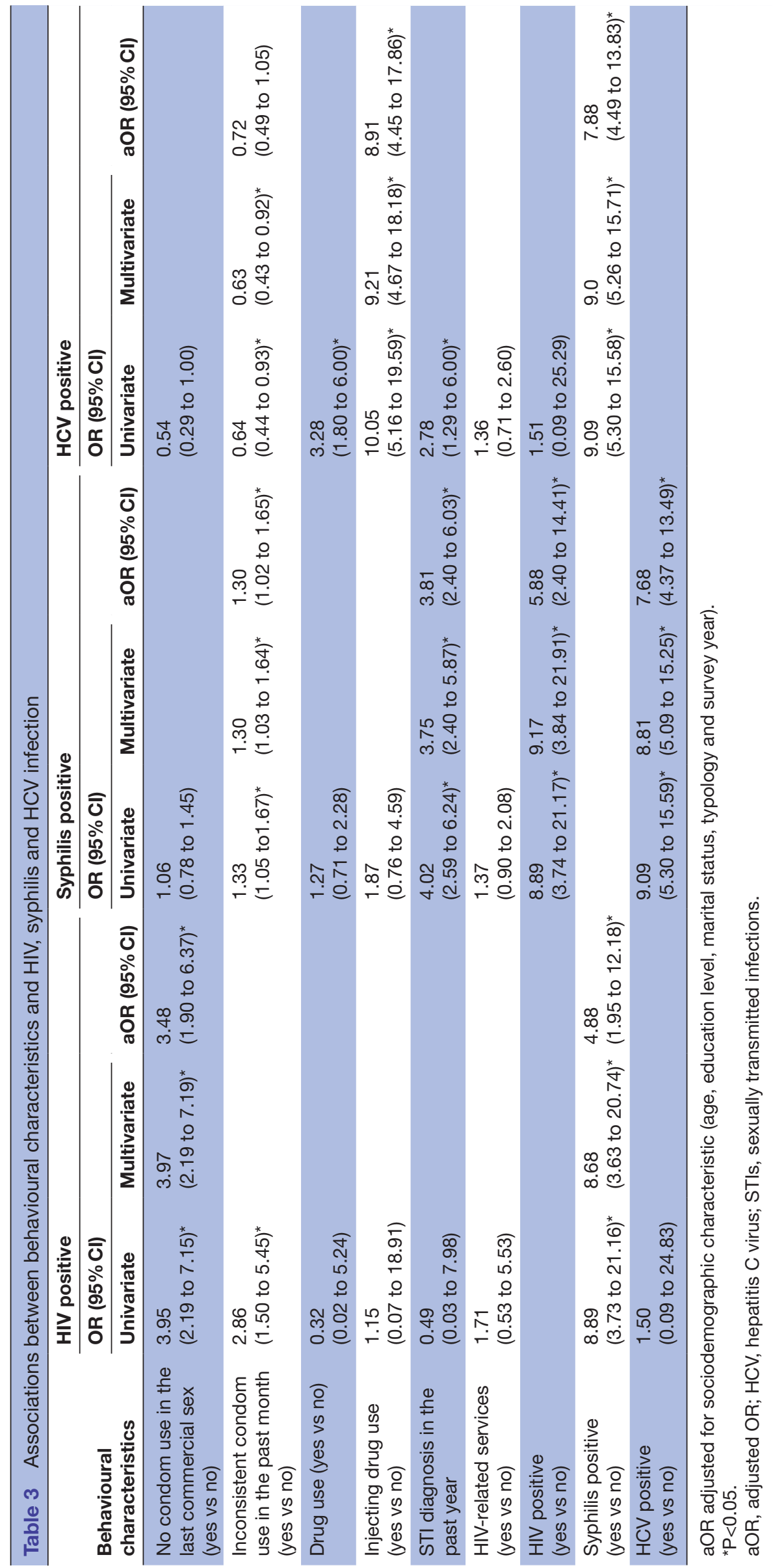


among Chinese FSWs in $2014^{9}$ but much higher than China's current estimated national HIV prevalence among the general population $(0.0598 \%) .{ }^{29}$ The prevalence of HIV among FSWs in Chongqing is higher than that in most provinces such as Beijing, ${ }^{30}$ Liaoning $^{31}$ and Hainan, ${ }^{32}$ but lower than that in Sichuan, ${ }^{33}$ Yunnan ${ }^{34}$ and Guangxi. ${ }^{35} \mathrm{HCV}$ prevalence fluctuated between 2013 and 2018 , with a 6 -year average of $0.72 \%$, but no significant trend was found. This result was in concordance with that of a previous study conducted in Guangxi. ${ }^{35}$ However, a previous study using national data reported that from 2008 to 2012, the overall HIV and HCV prevalence among FSWs in mainland China showed a decreasing trend ${ }^{13}$; the differences may be due to the differences in scope of study area and study period.

The results also indicated a worrying upward trend of syphilis infection (from $1.11 \%$ in 2013 to $2.00 \%$ in 2018), particularly among low-tier and middle-tier FSWs. As the third most prevalent notifiable infectious disease in categories A and B in China, syphilis has been on the rise since the 1990s. ${ }^{36}$ The reported total syphilis rate in China increased from 0.2/100 000 in 1993 to $32.86 / 100000$ in $2013 .^{36}$ The reported incidence of syphilis in Chongqing also showed an upward trend, with a reported incidence of 40.38/100 000 in $2014 .{ }^{37}$ However, researchers found that the increase in reported cases of syphilis in Chongqing in recent years may be related to large-scale syphilis screening by medical institutions, training on national standards for syphilis diagnosis and reporting, and laboratory improvement. ${ }^{38}$ The increase in syphilis prevalence among FSWs may also be related to the strengthening of syphilis detection, but it still needs attention. In agreement with previous studies, the FSWs infected with syphilis have a higher risk of HIV or HCV infection, and HIV or HCV infection is a risk factor of syphilis infection..$^{35}$ FSWs with syphilis were nearly five times more likely to be infected with HIV, and seven times more likely to be infected with HCV than FSWs without syphilis. This phenomenon may be due to their shared transmission routes, co-risk factors and adverse interaction. ${ }^{39}$ Thus, efforts should be increased to strengthen the comprehensive and combined intervention of AIDS, syphilis and other STIs for key groups.

During the study period, the proportion of FSWs who used condoms increased slightly, and condom use was associated with decreased HIV or syphilis infection risk. CCU is one of the most effective ways to prevent HIV infection, and its protection rate can reach $80 \%$ based on studies of persons during heterosexual sex with an HIVpositive partner. ${ }^{40}$ The $100 \%$ Condom Use Programme has been implemented in Chongqing since 2006 and has shown a promotional effect. The number of FSWs who practiced CCU increased. However, in 2018, only half of FSWs reported CCU in the last month. This proportion was lower than that in Liaoning and Hainan. ${ }^{31}{ }^{32}$ Previous studies indicated that clients' low support and negative norms towards condom use may be the main reason for the inconsistent condom use of FSWs. ${ }^{41-43}$ FSWs often compromise with their clients on condom use to earn money or establish a closer long-term relationship with clients. Thus, in addition to raising FSWs' awareness regarding HIV/STI prevention and improving their skills to negotiate condom use, promoting HIV intervention programmes targeting clients of FSWs and others is also essential.

No significant increasing or decreasing trend in drug use was observed among FSWs. The average prevalence of drug use was $3.3 \%$, while the national prevalence among FSWs was reported at $1.2 \% .^{13}$ The prevalence of injecting drug use seemed to be declining during the study period. Injecting drug use may exacerbate unsafe sexual behaviour and structural risks, leading to increased risk of HIV and other STIs. ${ }^{44}$ In 2017, the detection rates of HIV, syphilis and HCV among drug users in Chongqing were $5.4 \%, 5.9 \%$ and $44.9 \%$, respectively, much higher than those among the general population. ${ }^{46}$ FSWs who inject drugs are exposed to sexual and blood transmission routes and at a higher risk of contracting HIV and syphilis than other FSWs and injecting drug users. ${ }^{35} 47$ Similar to previous studies, the present study showed a strong association between injecting drug use and HCV infection, ${ }^{48}$ and sharing of needles may be the main and underlying route of HCV transmission. Drugs and prostitution involve relevant laws and ethics. Thus, HIV prevention programmes and outreach for FSWs may require enhanced thinking and increased attention to the individual's external risk environment and risk factors.

In addition, as previous studies have shown, lower-tier FSWs bear a heavier burden of HIV, syphilis and HCV than higher-tier FSWs, ${ }^{13}$ which may be attributed to the sociodemographic characteristics of lower-tier FSWs and their clients. It's found that low-tier FSWs generally have older age and lower education, which are often associated with a higher prevalence of HIV and syphilis. ${ }^{49}$ Low-tier FSWs with low education probably had insufficient knowledge about those diseases, less awareness about sex health and poor communication skills for condom use. ${ }^{50}$ And they may be serving more clients who also had low education, had low awareness and were unwilling to use a condom, particularly elderly man. ${ }^{17} 35$ Thus, future intervention programmes should be specified and targeted based on the needs of FSWs under different tiers, and pay more attention to low-tier FSWs and their clients. In addition, sex work is illegal and highly stigmatised in China; thus, FSWs face several barriers, such as fear of being arrested, fear of discrimination and awkwardness, to participant in AIDS-related services. ${ }^{51}$ AIDS intervention services must further cooperate with non-governmental organisations, increase condom promotion and jointly increase awareness of other STIs such as syphilis.

This study had several limitations. First, the data we used were based on serial cross-sectional surveillance surveys; therefore, trends of HIV, syphilis and HCV infection without exact reasons behind the changes were possibly found. Second, the survey was anonymous. Respondents who participated in a cross-sectional survey 
in a certain year may also participate in subsequent crosssectional surveys. However, estimating the proportion of FSWs who repeatedly participated in this study was not possible. Third, the data were collected mainly through self-reported questionnaires, which raise concerns about recall bias, reporting bias and social desirability bias. For example, respondents may conceal the truth when answering sensitive questions, such as drug use, which is illegal in China.

\section{CONCLUSIONS}

In conclusion, the HIV and HCV prevalence among FSWs in Chongqing remained stable during the study period, but syphilis had an upward trend among FSWs in low-tier and middle-tier venues. Although factors such as knowledge, condom use, injecting drug use and STI diagnosis were improved, nearly half of FSWs used condom inconsistently. Thus, more effective interventions for promoting CCU among FSWs and their clients are needed to reduce the spread of HIV/STIs, and more attention must be paid to low-tier and middle-tier FSWs.

Acknowledgements The authors express their thanks to the individuals who volunteered to participate in this study and Chinese Center for Disease Control and Prevention, Chongqing, China for their contributions to the study.

Contributors GW, RL and MY designed and coordinated the study. GW and RL was involved in the design and conducting of the survey. LH and MY analysed the data. $\mathrm{LH}, \mathrm{HZ}$, DJ and HQ participated in the interpretation of results. LH wrote the first draft of the manuscript. LH, HQ, HZ, DJ, GW, RL and MY revised the manuscript. All authors have read and approved the manuscript.

Funding This research was funded by the Chongqing Science and Technology Commission of the People's Republic of China in 2017 (Grant NO: cstc2017jcyjAX0185) and Chongqing Yuzhong District Science and Technology Bureau (20190116).

Map disclaimer The depiction of boundaries on this map does not imply the expression of any opinion whatsoever on the part of BMJ (or any member of its group) concerning the legal status of any country, territory, jurisdiction or area or of its authorities. This map is provided without any warranty of any kind, either express or implied.

Competing interests None declared.

Patient and public involvement Patients and/or the public were not involved in the design, or conduct, or reporting, or dissemination plans of this research.

Patient consent for publication Not required.

Ethics approval This survey was reviewed and approved by the Institutional Review Board of the National Center for AIDS Prevention and Control (NCAIDS), Center for Disease Control (CDC), China, and the medical ethics committee of Chongqing Medical University (IRB number:2017016).

Provenance and peer review Not commissioned; externally peer reviewed.

Data availability statement № data are available.

Open access This is an open access article distributed in accordance with the Creative Commons Attribution Non Commercial (CC BY-NC 4.0) license, which permits others to distribute, remix, adapt, build upon this work noncommercially, and license their derivative works on different terms, provided the original work is properly cited, appropriate credit is given, any changes made indicated, and the use is non-commercial. See: http://creativecommons.org/ licenses/by-nc/4.0/.

ORCID iD

Mengliang Ye http://orcid.org/0000-0001-9394-8682
REFERENCES

1 Shannon K, Crago A-L, Baral SD, et al. The global response and unmet actions for HIV and sex workers. Lancet 2018;392:698-710.

2 WHO. Report on global sexually transmitted infection surveillance, 2018, 2018. Available: https://www.who.int/reproductivehealth/ publications/stis-surveillance-2018/en/

3 Gil VE, Wang MS, Anderson AF, et al. Prostitutes, prostitution and STD/HIV transmission in mainland China. Soc Sci Med 1996;42:141-52.

4 Hong Y, Li X. HIV/AIDS behavioral interventions in China: a literature review and recommendation for future research. AIDS Behav 2009;13:603-13.

5 Hail-Jares K, Chang RCF, Choi S, et al. Intimate-partner and clientinitiated violence among female street-based sex workers in China: does a support network help? PLoS One 2015;10:e0139161.

6 Wang L, Wang N, Wang L, et al. The 2007 estimates for people at risk for and living with HIV in China: progress and challenges. $J$ Acquir Immune Defic Syndr 2009;50:414-8.

7 Pan S, Parish WL, Huang Y. Clients of female sex workers: a population-based survey of China. J Infect Dis 2011;204:S1211-7.

8 Su S, Chow EPF, Muessig KE, et al. Sustained high prevalence of viral hepatitis and sexually transmissible infections among female sex workers in China: a systematic review and meta-analysis. BMC Infect Dis 2016;16:2.

9 Tucker JD, Henderson GE, Wang TF, et al. Surplus men, sex work, and the spread of HIV in China. AIDS 2005;19:539-47.

$10 \mathrm{He} \mathrm{N}$. Emerging changes and characteristics of the HIV epidemic in China. Shanghai J Prev Med 2019;31:963-7.

11 CDC bureau. New progress in AIDS prevention and control in China in 2019, 2019. Available: http://www.nhc.gov.cn/jkj/s3586/201911/ c2388ce70bdd404ea6dfcd886591784d.shtml [Accessed $30 \mathrm{Nov}$ 2019].

12 Poon AN, Li Z, Wang N, et al. Review of HIV and other sexually transmitted infections among female sex workers in China. AIDS Care 2011;23:5-25.

13 Wang L, Tang W, Wang L, et al. The HIV, syphilis, and HCV epidemics among female sex workers in China: results from a serial cross-sectional study between 2008 and 2012. Clin Infect Dis 2014;59:e1-9.

14 Zhang H, Hsieh E, Wang L, et al. HIV/AIDS among female sex workers in China: epidemiology and recent prevention strategies. Curr HIVIAIDS Rep 2020;17:151-60.

15 Liang J, Deng W, Huang S, et al. Estimation of the population size of female sex workers in Jiangmen with enumeration and census methods. J Diagnosis Ther Dermato-venereology 2012;19:50-2.

16 Sun K, Jing Z, Yang Q, et al. Population size of female sex workers estimated by census method in the urban areas of Mengzi and Jianshui County, Honghe Prefecture. Chinese J AIDS STD 2019;25:294-6.

17 Chen X-S, Liang G-J, Wang Q-Q, et al. HIV prevalence varies between female sex workers from different types of venues in southern China. Sex Transm Dis 2012;39:868-70.

18 National Health and Family Planning Commission of the People's Republic of China. 2015 China AIDS response progress report, 2015. Available: http://www.unaids.org/sites/default/files/country/ documents/CHN_narrative_report_2015.pdf

19 Jia Y, Lu F, Sun X, et al. Sources of data for improved surveillance of HIV/AIDS in China. Southeast Asian J Trop Med Public Health 2007;38:1041-52.

20 Wang L, Wang N. HIV/AIDS epidemic and the development of comprehensive surveillance system in China with challenges. Chin Med J 2010;123:3495-500.

21 Sun X, Wang N, Li D, et al. The development of HIV/AIDS surveillance in China. AIDS 2007;21:S33-8.

22 Lin W, Chen S, Seguy N, et al. Is the HIV sentinel surveillance system adequate in China? findings from an evaluation of the National HIV sentinel surveillance system. Western Pac Surveill Response $J$ 2012;3:61-8.

23 Ghys PD, Jenkins C, Pisani E. Hiv surveillance among female sex workers. AIDS 2001;15:S33-40.

24 Li P, Li D, Ge L, et al. Prevalence of HIV infection, syphilis and hepatitis C in female sex workers in China, 2010-2015. Dis Surveill 2017;32:287-91.

25 Guo W, Bao S, Lin W, et al. Estimating the size of HIV key affected populations in Chongqing, China, using the network scale-up method. PLoS One 2013;8:e71796.

26 Ma D, Zhang Y, Zhou L, et al. Epidemic analysis on high-risk HIV/ AIDS population in Chongqing from 2006 to 2012. J Third Mil Med Univ 2015;37:689-92. 
27 Yu H, Zhang W, Lu R, et al. Epidemiological characteristics and secular trend of HIV infection and AIDS in Chongqing during 1995 and 2015. J Third Mil Med Univ 2017;39:2051-8.

28 National Center for STD/AIDS Prevention and Control, Chinese Center for Disease Control and Prevention. Operational manual for the implementation program of national AIDS sentinel surveillance, 2012.

29 Huang MB, Ye L, Liang BY, et al. Characterizing the HIV/AIDS epidemic in the United States and China. Int J Environ Res Public Health 2016;13.

30 Sun Y, Li G, Lu H. Characteristics of female sex workers and their HIV and syphilis prevalence in Beijing, 2011-2016. Chinese J AIDS STD 2019;25:260-3.

31 Sun L, Zhou D, Jiang F, et al. Ananalysis of HIV / AIDS sentinel surveillance to high risk populations in Liaoning Province from 2012 to 2014. Chinese J Dis Control Prev 2015;19:1227-30.

32 Zeng X, Wang Z, Nie S. Survey on AIDS related knowledge, behaviors and infectious conditions among female sex workers in Hainan during 2011-2013. China Trop Med 2015;15:1207-9.

33 Yang H, Su L, Ye L, et al. [Incidence rates of HIV-1 epidemics among 4 high risk groups in Sichuan province during 2011-2015]. Zhonghua Liu Xing Bing Xue Za Zhi 2017;38:1649-54.

34 Wang J, Jia $\mathrm{M}$, Luo $\mathrm{H}$, et al. [Analysis of the risky behaviors among HIV positive female sex workers]. Zhonghua Yu Fang Yi Xue Za Zhi 2015;49:978-82.

35 Chen Y, Shen Z, Morano JP, et al. Bridging the epidemic: a comprehensive analysis of prevalence and correlates of HIV, hepatitis $\mathrm{C}$, and syphilis, and infection among female sex workers in Guangxi Province, China. PLoS One 2015;10:e0115311.

36 Gong X, Yue X, Teng F, et al. Syphilis in China from 2000 to 2013: epidemiological trends and characteristics. Chinese J Dermatology 2014;47:310-5.

37 Jiang $\mathrm{H}$, Guo X, Chen $\mathrm{Y}$. Prevalence of sexually transmitted diseases in Chongqing municipality,2007-2014. Chinese J Public Heal 2016;32:1556-60.

38 Guo X, Jiang H, Wu G, et al. Investigation and analysis on influencing factors of syphilis and gonorrhoea epidemic report in Chongqing during 2010-2014. Lab Med Clin 2019;16:2144-6.

39 Shimelis T, Lemma K, Ambachew H, et al. Syphilis among people with HIV infection in southern Ethiopia: sero-prevalence and risk factors. BMC Infect Dis 2015;15:189.
40 Weller S, Davis K, Davis-Beaty K. Condom effectiveness in reducing heterosexual HIV transmission. Cochrane Database Syst Rev 2002:CD003255.

$41 \mathrm{Ma}$ Q, Jiang J, Pan X, et al. Consistent condom use and its correlates among female sex workers at hair salons: a cross-sectional study in Zhejiang Province, China. BMC Public Health 2017;17:910.

42 Hao C, Guida J, Morisky DE, et al. Family network, workplace network, and their influence on condom use: a qualitative study among older female sex workers in China. J Sex Res 2015;52:924-35.

43 Jie W, Xiaolan Z, Ciyong L, et al. A qualitative exploration of barriers to condom use among female sex workers in China. PLoS One 2012;7:e46786.

44 Wirtz AL, Peryshkina A, Mogilniy V, et al. Current and recent drug use intensifies sexual and structural HIV risk outcomes among female sex workers in the Russian Federation. Int J Drug Policy 2015;26:755-63.

45 Ulibarri MD, Strathdee SA, Ulloa EC, et al. Injection drug use as a mediator between client-perpetrated abuse and HIV status among female sex workers in two Mexico-US border cities. AIDS Behav 2011;15:179-85.

46 Jiang H, Lu R, Wu G. Analysis of AIDS sentinel surveillance results among drug users in Chongqing from 2010 to 2017. Mod Prev Med 2019;46:1883-6.

47 Hail-Jares K, Choi S, Duo L, et al. Occupational and demographic factors associated with drug use among female sex workers at the China-Myanmar border. Drug Alcohol Depend 2016;161:42-9.

48 Goldenberg SM, Montaner J, Braschel M, et al. Dual sexual and drug-related predictors of hepatitis $C$ incidence among sex workers in a Canadian setting: gaps and opportunities for scale-up of hepatitis $C$ virus prevention, treatment, and care. Int $J$ Infect Dis 2017;55:31-7.

49 Chen X-S, Wang Q-Q, Yin Y-P, et al. Prevalence of syphilis infection in different tiers of female sex workers in China: implications for surveillance and interventions. BMC Infect Dis 2012;12:84.

50 Zeng $\mathrm{H}$, Zhao $\mathrm{Y}$, Meng $\mathrm{S}$, et al. Exploring HIV prevention strategies among street-based female sex workers in Chongqing, China. Int Environ Res Public Health 2015;12:855-70.

51 Zeng $\mathrm{H}$, Zhang $\mathrm{L}$, Zhao $\mathrm{Y}$, et al. HIV prevention among street-based sex workers (SSWs) in Chongqing, China: interviews with SSWs, clients and healthcare providers. Health Soc Care Community 2016;24:e173-80. 